Research Article

\title{
Preparation of Graphene Oxide and Its Application as Substrates for SERS
}

\author{
Xiaodong Li (i) \\ Key Laboratory of Songliao Aquatic Environment, Ministry of Education, Jilin Jianzhu University, Changchun 130118, China \\ Correspondence should be addressed to Xiaodong Li; 1xdjlju@163.com
}

Received 3 July 2018; Accepted 23 September 2018; Published 17 October 2018

Academic Editor: Carola Esposito Corcione

Copyright (c) 2018 Xiaodong Li. This is an open access article distributed under the Creative Commons Attribution License, which permits unrestricted use, distribution, and reproduction in any medium, provided the original work is properly cited.

Graphene oxide (GO) was synthesized by a modified Hummer's method and was then reduced by the hydrothermal process. Both GO and reduced GO (rGO) were employed as surface-enhanced Raman scattering (SERS) substrates to detect rhodamine 6G (R6G). After adsorbed on the surface of GO, the fluorescence background of R6G was highly quenched, and its Raman scatterings were enhanced. When adsorbed on the surface of $\mathrm{rGO}$, the fluorescence background was further quenched at the price of lower SERS intensity. The results displayed that oxygen groups on the surface of GO had positive effect on the SERS effect of GO. The amount of oxygen groups on the surface of GO should be one key parameter to adjust the SERS activity of GO.

\section{Introduction}

Surface-enhanced Raman spectroscopy (SERS) has attracted much attention since its discovery in the 1970s [1]. It can increase the Raman scattering signal of probe molecules near or adsorbed on SERS substrates (e.g., gold and silver) by several orders of magnitude through electromagnetic enhancement mechanism (EM) and chemical enhancement mechanism (CM) and can even achieve single molecular detection level. Commonly, the EM provides the majority of the SERS enhancement effect and CM contributes to the minor portions. Moreover, the CM and EM are often associated with each other; thus, less research studies on the aspect of CM were carried out. Therefore, it is very important to look for a new type of SERS substrate that only supports CM.

Graphene is a two-dimensional film material that is formed by stacking single-layer carbon atoms in a hexagonal grid structure [2]. It has unique electrical, mechanical, optical, and magnetic properties, and thus has huge potential applications in the field of biosensors [3] and electrochemical sensors $[4,5]$, biocompatible drug transport [6], fuel cell electrocatalysis [7], energy storage [8,9], etc. In the year of 2009, Xie et al. [10] discovered that graphene can quench the fluorescence of R6G molecules adsorbed on its surface and enhanced the Raman scattering of R6G through $\mathrm{CM}$, making graphene a potential SERS-active substrate. Graphene has many unique properties compared to conventional SERS-active substrates (e.g., gold and silver): (1) atomic-scale smooth surface, (2) high light transmission in the visible spectral range (single-layer graphite: the light transmittance of the alkene is as high as 95\%), and (3) the surface plasmon resonance (SPR) response is located in the terahertz region, which is far away from the visible light spectral region, and its SPR is not excited during SERS detection (e.g., using a $514.5 \mathrm{~nm}$ laser). Therefore, under the conventional SERS detection conditions, graphene does not support the EM and can only enhance the Raman signal of the probe molecule through $\mathrm{CM}$. In this way, graphene can be an ideal SERS substrate to investigate the CM for SERS [11].

Graphene oxide (GO) has also received extensive attentions as an important derivative of graphene. Compared with graphene, GOs are modified with a large number of oxygen-containing groups, such as hydroxyl, carboxyl, and epoxy, on the surface and edge regions. It has been found that GO displayed certain SERS activity as well, and its SERS activity is related to its surface-modified oxygen-containing groups [12]. Herein, the effect of the number of oxygencontaining groups in GO on its SERS activity was studied. 
GO was prepared by Hummers' method and was then reduced by a hydrothermal process to remove part of oxygencontaining groups of GO. Using rhodamine 6G (R6G) as a probe molecule, we studied the SERS activities of GO before and after the reduction process to study the effect of number of oxygen-containing groups on the SERS activities of $\mathrm{GO}$.

\section{Experimental}

2.1. Materials. All reagents were of analytical grade and used as purchased without further purification. Natural graphite (99 wt. \%) was purchased from Qingdao Yingshida Graphite Co., Ltd. Concentrated sulfuric acid (98 wt. \%), $\mathrm{KMnO}_{4}$ (99.5\%), $\mathrm{H}_{2} \mathrm{O}_{2}$ (30 wt. \%), $\mathrm{HCl}$ (37 wt. \%), and $\mathrm{NaOH}(96 \%)$ were purchased from Beijing Chemical Works. $\mathrm{NaNO}_{3}$ (99.0\%) was obtained from Tianjin Guangfu Chemical Reagent Co., Ltd. Rhodamine 6G (R6G) was obtained from Waldeck GmbH \& Co. KG. MilliQ water (18.6 M $\Omega$ ) was used to prepare aqueous solutions.

\subsection{Experimental Process}

2.2.1. Preparation of GO. Hummers' method was used to prepare GO. The reaction was carried out in an Erlenmeyer flask of $500 \mathrm{~mL}$ in capacity. Typically, graphite powder $(2 \mathrm{~g})$ was added into the mixture of $\mathrm{NaNO}_{3}(1.5 \mathrm{~g})$ and concentrated sulfuric acid $(67.5 \mathrm{~mL})$ under magnetic stirring. Then, $\mathrm{KMnO}_{4}(9 \mathrm{~g})$ was gradually added into the mixture in an ice bath that the reaction temperature was strictly controlled below $10^{\circ} \mathrm{C}$. The addition process was allowed to complete in 1 hour. The mixture was stirred for another 2 hours in the ice bath and for 5 days at room temperature. Then, sulfuric acid solution $(200 \mathrm{~mL}, 5 \mathrm{wt}$. \%) was used to dilute the obtained suspensions with $2 \mathrm{~h}$ mild stirring. $\mathrm{H}_{2} \mathrm{O}_{2}(6 \mathrm{~mL})$ was slowly added to the suspensions, and the suspensions were turned into bright yellow in color. After another $2 \mathrm{~h}$ stirring, the reaction was completed. The suspensions were centrifuged and washed with $1000 \mathrm{~mL}$ of mixed solution of $\mathrm{H}_{2} \mathrm{SO}_{4}$ (3 wt. $\%)$ and $\mathrm{H}_{2} \mathrm{O}_{2}(0.5$ wt. \%). Finally, the obtained product was then washed repeatedly with pure water until it became neutral in the $\mathrm{pH}$ value.

2.2.2. Reduction of GO. GO was reduced by a hydrothermal method. The upper layer of GO suspension solution was collected and sonicated for $10 \mathrm{~min}$ at $50 \mathrm{~W}$. Then, $40 \mathrm{~mL}$ of the suspension solution was poured into the reactor, and the $\mathrm{pH}$ of solution was adjusted to 5 with $\mathrm{NaOH}$ aqueous solution $(2 \mathrm{~mol} / \mathrm{L})$. The reactor was then placed in an oven, the reaction temperature was $180^{\circ} \mathrm{C}$, and the reaction time was set to 10 and 30 minutes.

2.3. Characterization. X-ray diffraction (XRD) patterns were recorded on a D8 ADVANCE diffractometer using $\mathrm{Cu} \mathrm{K} \alpha$ line as the source $(\lambda=1.5418 \AA$ and operating at $30 \mathrm{kV}$ and $20 \mathrm{~mA}$ ). Fourier transform infrared (FT-IR) spectra were recorded with $\mathrm{KBr}$ pellets, using a Bruker Vertex-70 FT-IR spectrophotometer to characterize the framework of the sample (the typical pellet consists of $1 \mathrm{wt}$. $\%$ sample and $99 \mathrm{wt}$. \% $\mathrm{KBr}$ ). The Raman spectra were recorded using a Renishaw 1000 microspectrometer connected to a Leica microscope with an objective lens of $50 \times(\mathrm{NA}=0.5)$. The spectra were collected with the laser power of $0.33 \mathrm{~mW}$, with the accumulation time of $10 \mathrm{~s}$, and with the excitation wavelength of $514.5 \mathrm{~nm}$. Scanning electron microscopy (SEM) images were taken on a PHILIPS XL30 field-emission environmental scanning electron microscope (Netherlands) instrument. Atomic force microscopy (AFM) images were obtained in the tapping mode in air with ScanAsyst (Dimension Icon, Veeco Instruments/Bruker, Germany).

\section{Results and Discussion}

3.1. Structural and Morphological Characterization of GO. Figure 1 shows the XRD spectra of graphite powder and GO. In the XRD spectrum of graphite, a strong characteristic diffraction peak appears near $26.5^{\circ}$ to $2 \theta$, which is attributed to high-purity graphite, corresponding to the (002) plane with the spacing $d=0.34 \mathrm{~nm}$. This characteristic peak indicates that the untreated graphite powder has a regular spatial arrangement. In the XRD spectrum of GO, the diffraction peak originally assigned to the (002) plane of graphite disappeared, and a new characteristic peak appears near $2 \theta$ of $11.6^{\circ}$, which belongs to the diffraction peak of the GO (001) plane. The intensity of the diffraction peak is much weaker than that of the graphite powder, indicating the increase in the interlayer spacing of graphite. This is because the basal planes and edges of chemically treated graphite sheets are modified with oxygen-containing functional groups such as hydroxyl, carboxyl, and epoxy groups, which greatly increase the interlamellar spacing and destroy the original crystal structure of graphite. The disappearance of the (002) plane diffraction peak indicates that the original graphite structure was oxidized and ultrasonically exfoliated, and the size became smaller and the crystallinity became worse.

Figure 2(a) shows the surface morphology of GO observed using SEM. It can be seen that the obtained GO sheet has a lot of pleated structure and the edge area is very thin, indicating that the prepared sample is few-layer GO sheet. To further determine the thickness of the GO sheet, we performed AFM characterization of the prepared GO sample, as shown in Figure 2(b). It can be seen that the GO sheet has a thickness of about $1.0 \mathrm{~nm}$ and a width of several hundred nanometers. We know that the thickness of singlelayer graphene is $0.334 \mathrm{~nm}$. GO is an oxidized graphene sheet. There are a large number of oxygen-containing groups on the surface and in the edge region, which makes the thickness of GO increase from graphene to $0.7-1.2 \mathrm{~nm}$. Therefore, we believe that the GO obtained by our preparation is a single layer structure.

FT-IR spectrum was taken as well to investigate the chemical structure of GO sheets, as shown in Figure 3. A number of characteristic bands attributed to oxygen containing groups can be observed in the collected spectrum: a broad absorption band centered at $3392 \mathrm{~cm}^{-1}$ is attributed 


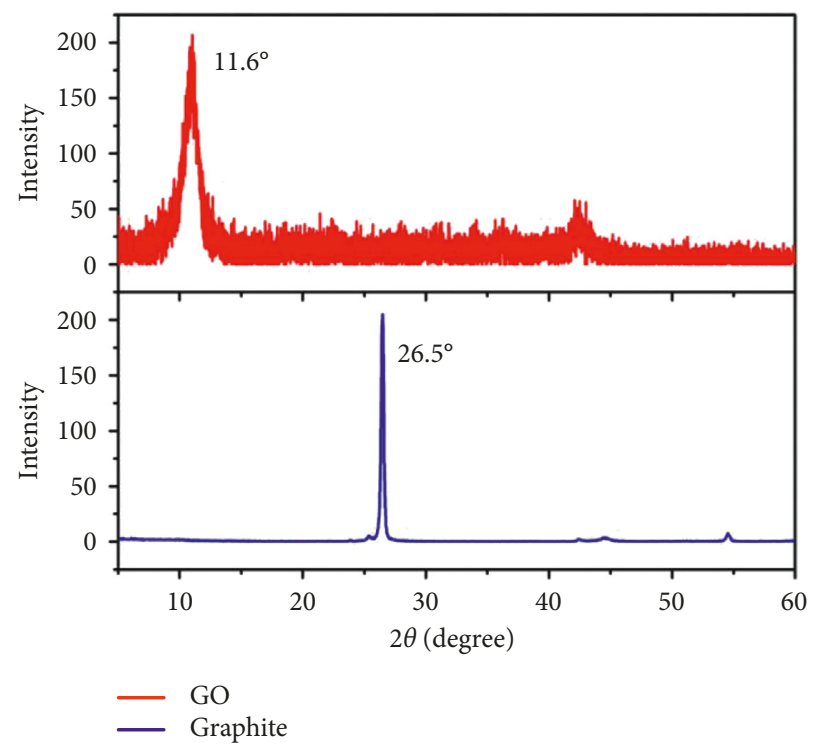

FIGURE 1: XRD patterns of graphite powder and GO.
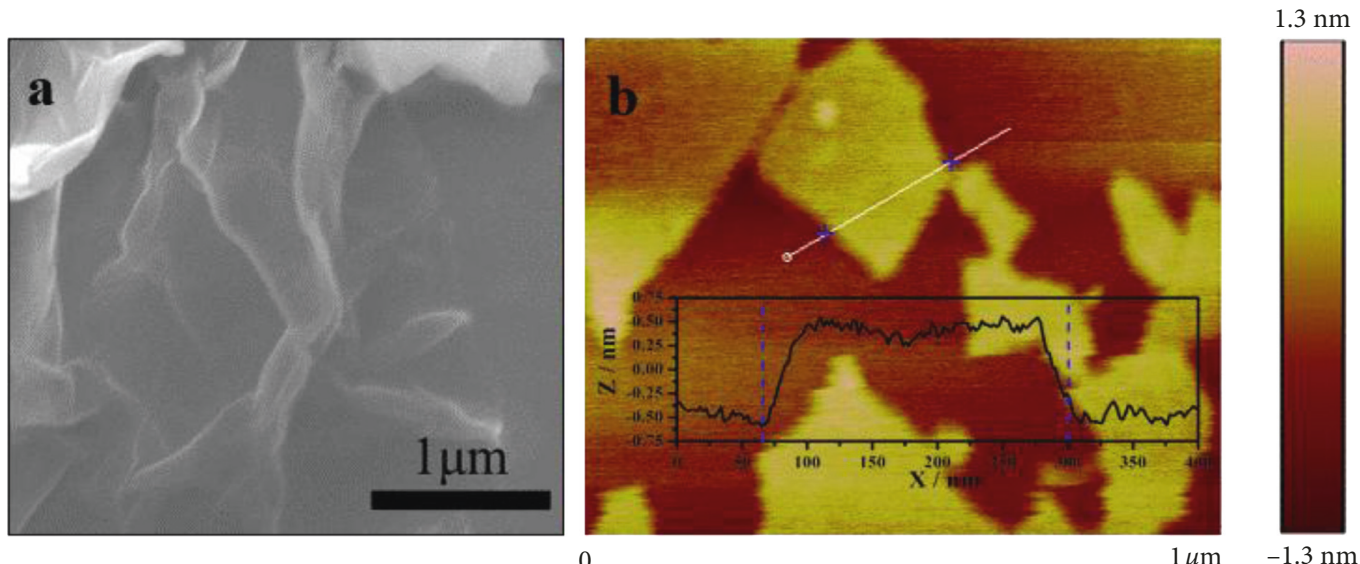

Figure 2: SEM (a) and AFM (b) images of GO.

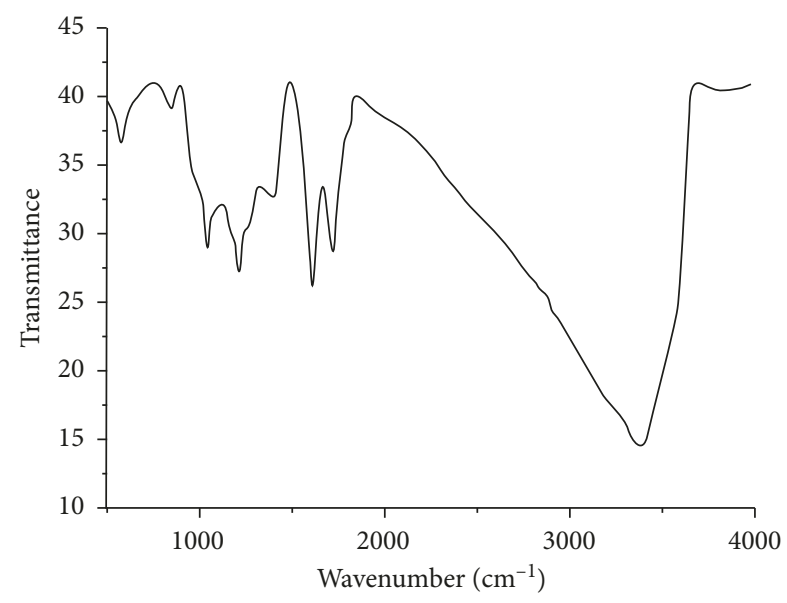

FIGURE 3: FT-IR spectrum of as-prepared GO. to the stretching vibration of $\mathrm{OH}$ from intercalated water, $-\mathrm{OH}$ and $-\mathrm{COOH}$ groups across $\mathrm{GO}$; the band centered at $1728 \mathrm{~cm}^{-1}$ is attributed to $\mathrm{C}=\mathrm{O}$ bond stretching vibrations in the carbonyl and carboxyl groups; the band centered at $1406 \mathrm{~cm}^{-1}$ is assigned to the bending vibration of the $\mathrm{OH}$ bond; the band centered at $1224 \mathrm{~cm}^{-1}$ is attributed to the stretching vibration of the $\mathrm{CO}$ bond in the epoxy group; and the band centered at $1053 \mathrm{~cm}^{-1}$ is attributed to the alkoxy group $\mathrm{C}=\mathrm{O}=\mathrm{C}$ bond stretching vibration. In addition, characteristic absorption peaks attributed to the $\mathrm{C}=\mathrm{C}$ bond also appear in the infrared absorption spectrum, which are located at around $1624 \mathrm{~cm}^{-1}$ and $870 \mathrm{~cm}^{-1}$ (weaker). The FTIR spectrum of $\mathrm{GO}$ confirms that the $\mathrm{GO}$ sheets are modified with several types of oxygen-containing groups, that is, oxygen $(-\mathrm{OH})$, carboxyl $(-\mathrm{COOH})$, carbonyl $(-\mathrm{C}=\mathrm{O})$, and epoxy (C-O-C). 
3.2. SERS Activity of GO. R6G, a commonly used dye molecule in SERS detection, was used as the probing molecule to investigate the SERS activity of GO. Figure 4 shows the Raman spectrum of R6G dripped on the surface of a silicon wafer and GO. Among them, the red line corresponds to the Raman spectrum of R6G dropped on the silicon wafer. We only observed a strong fluorescence background in this spectrum but failed to observe the characteristic Raman bands of R6G. This is because (1) an argon ion laser with the laser line of $514.5 \mathrm{~nm}$ was used for Raman detection. This laser irradiation condition can excite the fluorescence signal of the R6G molecule. (2) The fluorescence scattering cross section of the R6G molecule $\left(10^{-19} \mathrm{~cm}^{2}\right)$ is much larger than its Raman scattering cross section $\left(10^{-22} \mathrm{~cm}^{2}\right)$. This results in the weak Raman scattering signals of R6G that were annihilated under the strong fluorescence background, and no characteristic Raman peaks can be observed in its Raman spectrum.

When R6G is dripped onto the GO surface, we can observe the characteristic Raman peaks of the significantly enhanced R6G in its Raman spectrum, and the fluorescence background is also greatly attenuated. For example, the bands at around 1647,1507 , and $1360 \mathrm{~cm}^{-1}$ belong to the $\mathrm{C}-\mathrm{C}$ vibration of the benzene ring in the $\mathrm{R} 6 \mathrm{G}$ structure; the bands at around 1574 and $1309 \mathrm{~cm}^{-1}$ can be assigned to the in-plane bending vibrations of N-H; the 1187 and $1126 \mathrm{~cm}^{-1}$ bands are attributed to the in-plane vibration of $\mathrm{C}-\mathrm{O}-\mathrm{C}$; the peak at $773 \mathrm{~cm}^{-1}$ is attributed to the bending vibration of $\mathrm{C}-\mathrm{H}$; and the peak at $612 \mathrm{~cm}^{-1}$ is due to $\mathrm{C}-\mathrm{C}-\mathrm{C}$ ring in-plane bending mode, respectively. Moreover, the peak at $1596 \mathrm{~cm}^{-1}$ attributed to the G-band of GO and the peak at $521 \mathrm{~cm}^{-1}$ attributed to the silicon substrate can be observed in the Raman spectrum as well. The results show that GO has a certain SERS activity. This may be attributed to the following two aspects: (1) there is a large $\mathrm{sp}^{2}$ region in the GO sheet. The R6G molecule can be adsorbed on the surface through the $\pi-\pi$ interaction. The fluorescence signal from R6G can be annihilated energy transfer between R6G and GO. After the original strong fluorescent background is "weaken," the relatively weak Raman scattering signal "appears." (2) There are a large number of oxygencontaining groups in the structure of GO. The oxygen in these oxygen-containing groups can generate a small local electric field and amplify the Raman scattering signal of the R6G molecules around it.

In order to further determine the contribution of oxygen-containing groups to the SERS activity, a hydrothermal reduction process was performed to adjust reduction degree of $\mathrm{GO}$, which is directly relative to the number of oxygen-containing groups on GO sheet, by controlling the reaction time. Subsequently, GO and reduced graphene oxide (rGO) prepared by different reduction time (10,30 min) were used as substrates for SERS detection, and $\mathrm{R} 6 \mathrm{G}$ was used as the probing molecule to compare their SERS activities, as shown in Figure 5. It can be seen from the figure that the R6G molecule adsorbed on the GO surface has the strongest SERS signal, followed by the SERS signal on the rGO surface prepared after $10 \mathrm{~min}$ reduction, and the SERS signal on the rGO surface prepared after reduction for

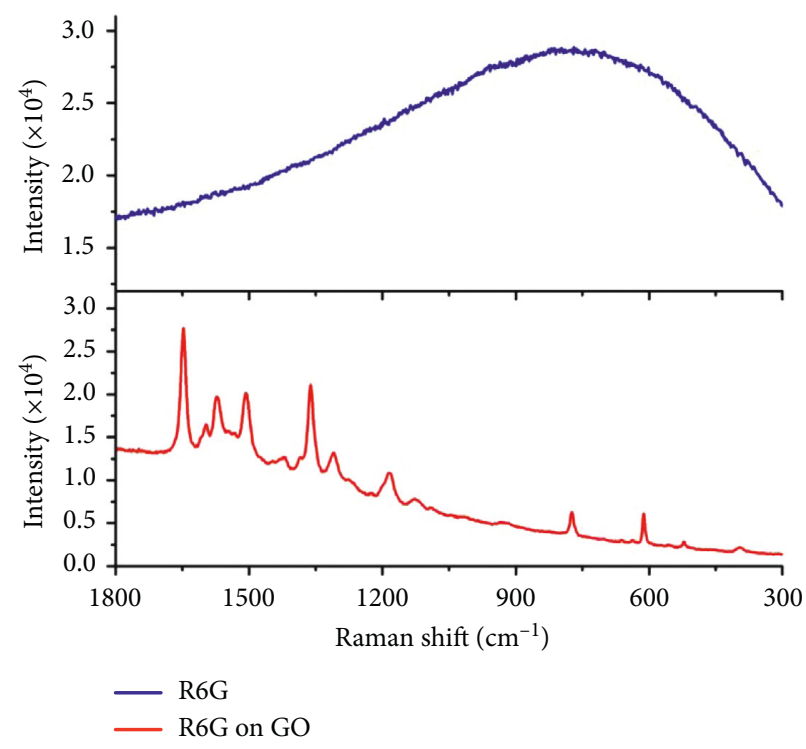

FIgURE 4: Raman spectra of R6G drop casted on the surface of Si wafer and GO. The inset displays the structure of R6G.

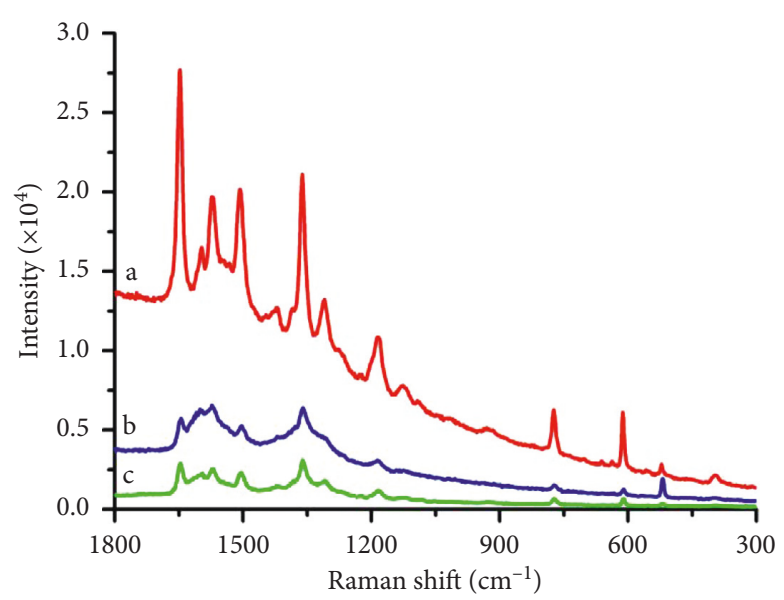

FIGURE 5: SERS spectra of R6G adsorbed on the surface of GO (a), rGO (10 $\mathrm{min})(\mathrm{b})$, and rGO (30 $\mathrm{min})(\mathrm{c})$, respectively.

$30 \mathrm{~min}$ is the weakest. The length of the reduction time is related to the degree and amount of oxygen-containing groups removed. The longer the reduction time, the more oxygen-containing groups are removed and the less oxygencontaining groups are left on the GO surface and edges. Therefore, the reduction of oxygen-containing groups in GO is related to the decrease in the SERS activity. In addition, we also noticed that the fluorescence background in the SERS spectrum gradually decreased with the prolonged reduction time. This may be because some of the $\mathrm{sp}^{2}$ structures were recovered during the reduction process, which increased the size of the $\mathrm{sp}^{2}$ structure and further improved the fluorescence quenching efficiency of the R6G molecules.

\section{Conclusions}

In summary, we prepared GO using Hummers' method and studied its SERS activity using R6G as a probe molecule. 
When the probe molecule is adsorbed on the GO surface, its fluorescent background is greatly attenuated and its Raman scattering is enhanced. In addition, after the hydrothermal reduction of GO, its SERS intensity has decreased, but the intensity ratio between signal and background has increased. This shows that the oxygen-containing group in GO has a certain influence on its SERS activity. It should be noted that although GO has strong SERS activity, its fluorescence background in the SERS spectrum is still relatively strong, and the intensity ratio between signal and background is not high. The moderate reduction can effectively increase the intensity ratio between the signal and the background, but at the expense of some signal strength. For this reason, we will further study how to balance the relationship between the two by controlling the reduction time to obtain better SERS activity.

\section{Data Availability}

The data used to support the findings of this study are included within the article.

\section{Conflicts of Interest}

The authors declare that they have no conflicts of interest.

\section{Acknowledgments}

This work was supported by the Natural Science Foundation of Jilin Province Department of Education (no. JJKH20170234KJ).

\section{References}

[1] M. Fleischm, P. J. Hendra, and A. McQuillan, "Raman spectra of pyridine adsorbed at a silver electrode," Chemical Physics Letters, vol. 26, no. 2, pp. 163-166, 1974.

[2] K. S. Novoselov, A. K. Geim, S. V. Morozov et al., "Electric field effect in atomically thin carbon films," Science, vol. 306, no. 5696, pp. 666-669, 2004.

[3] C. H. Lu, H. H. Yang, C. L. Zhu, X. Chen, and G. N. Chen, "A graphene platform for sensing biomolecules," Angewandte Chemie International Edition, vol. 48, no. 26, pp. 4785-4787, 2009.

[4] L. R. Li, H. G. Fu, and T. H. Lu, "Electrocatalytic performance of graphene supported Ir catalyst for ammonia oxidation," Chemical Journal of Chinese Universities, vol. 33, no. 1, pp. 102-106, 2012.

[5] C. L. He, C. L. Liu, T. P. Xie et al., "Preparation of graphene and electrocatalysis oxidation of p-hydroquinone at graphene modified electrode," Chemical Journal of Chinese Universities, vol. 33, no. 6, pp. 1290-1294, 2012.

[6] Z. Liu, J. T. Robinson, X. M. Sun, and H. Dai, "PEGylated nanographene oxide for delivery of water-insoluble cancer drugs," Journal of the American Chemical Society, vol. 130, no. 33, pp. 10876-10877, 2008.

[7] W. He, L. L. Zou, Y. Zhou et al., "In-situ synthesis of Pt nanoparticles on graphene nanosheets and its electrocatalysis for the oxygen reduction reaction," Chemical Journal of Chinese Universities, vol. 33, no. 1, pp. 133-138, 2012.
[8] L. Wang, K. Lee, Y. Y. Sun et al., "Graphene oxide as an ideal substrate for hydrogen storage," ACS Nano, vol. 3, no. 10, pp. 2995-3000, 2009.

[9] Z. L. Hu, M. Aizawa, Z. M. Wang, N. Yoshizawa, and H. Hatori, "Synthesis and characteristics of graphene oxidederived carbon nanosheet-Pd nanosized particle composites," Langmuir, vol. 26, no. 9, pp. 6681-6688, 2010.

[10] L. M. Xie, X. Ling, Y. Fang, J. Zhang, and Z. Liu, "Graphene as a substrate to suppress fluorescence in resonance raman spectroscopy," Journal of the American Chemical Society, vol. 131, no. 29, pp. 9890-9891, 2009.

[11] X. Ling, L. M. Xie, Y. Fang et al., "Can graphene be used as a substrate for raman enhancement?," Nano Letters, vol. 10, no. 2, pp. 553-561, 2010.

[12] X. X. Yu, H. B. Cai, W. H. Zhang et al., "Tuning chemical enhancement of SERS by controlling the chemical reduction of graphene oxide nanosheets," ACS Nano, vol. 5, no. 2, pp. 952-958, 2011. 

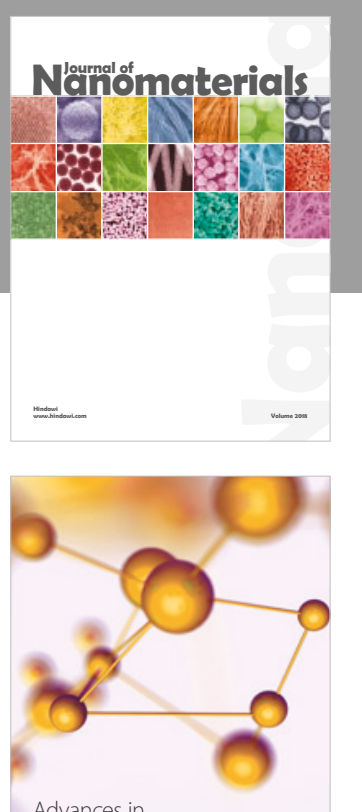

Physical Chemistry
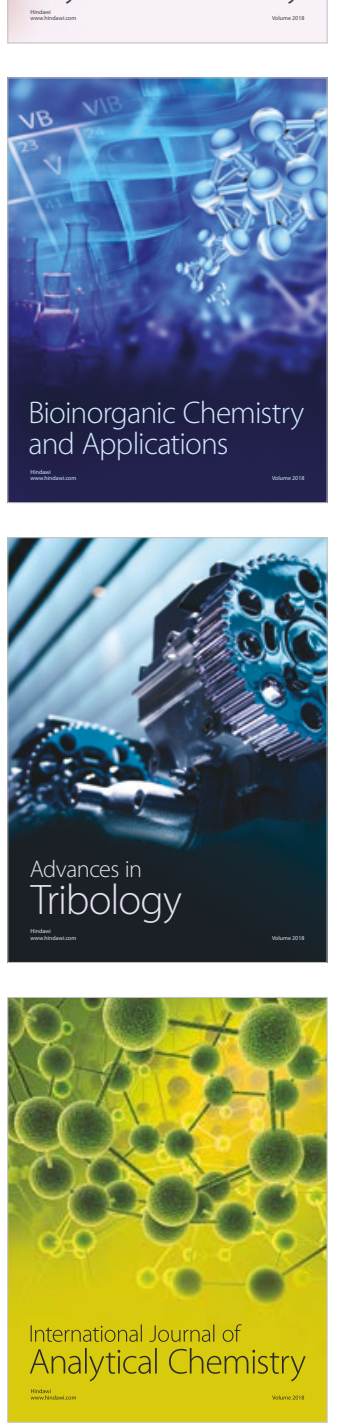

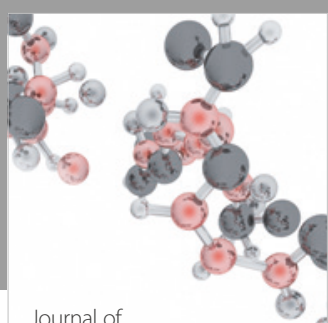

Analytical Methods

in Chemistry

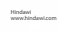

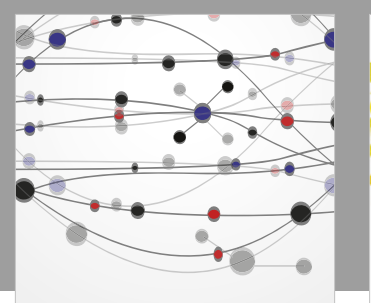

The Scientific World Journal

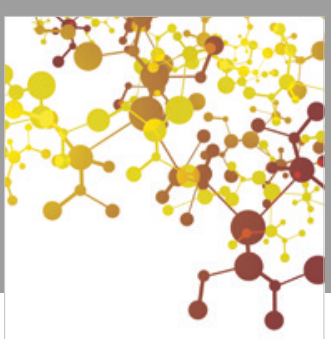

Journal of

Applied Chemistry
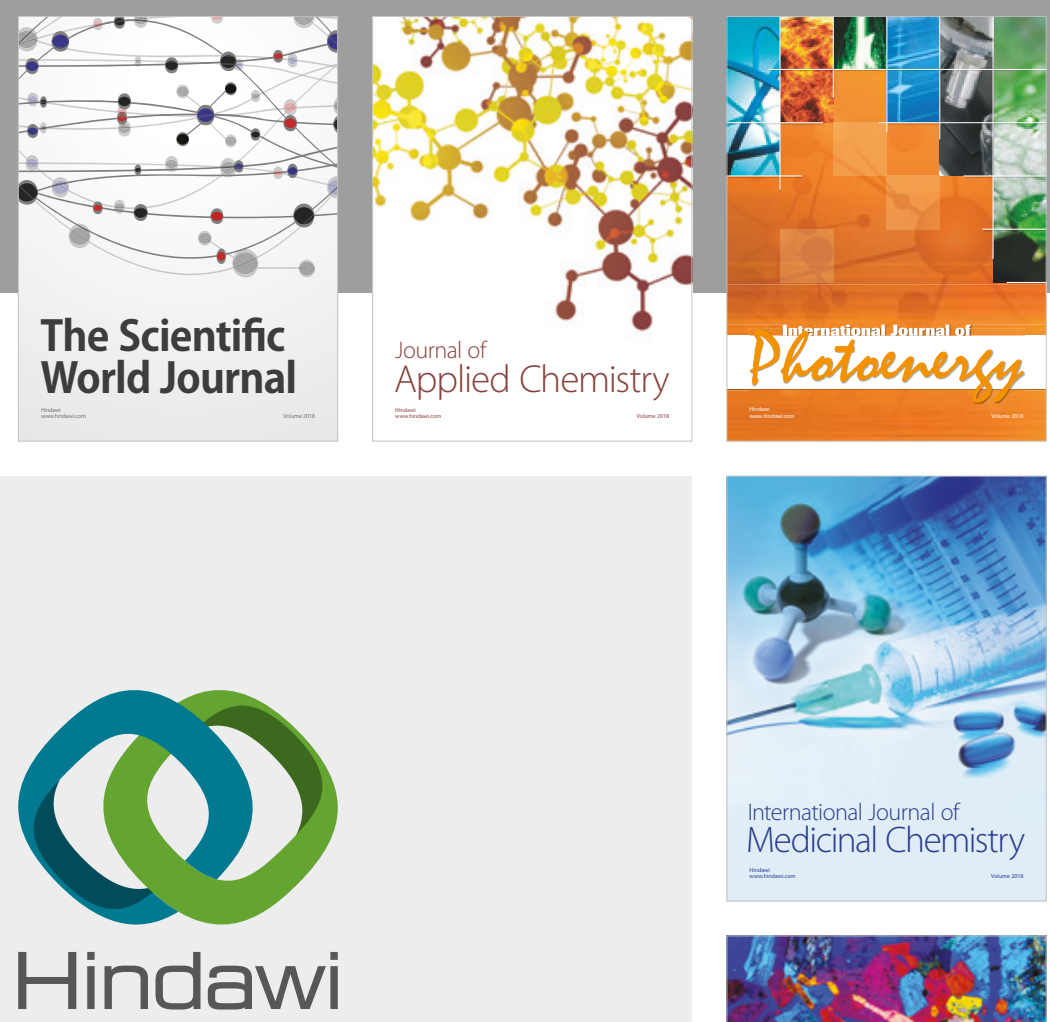

Submit your manuscripts at

www.hindawi.com
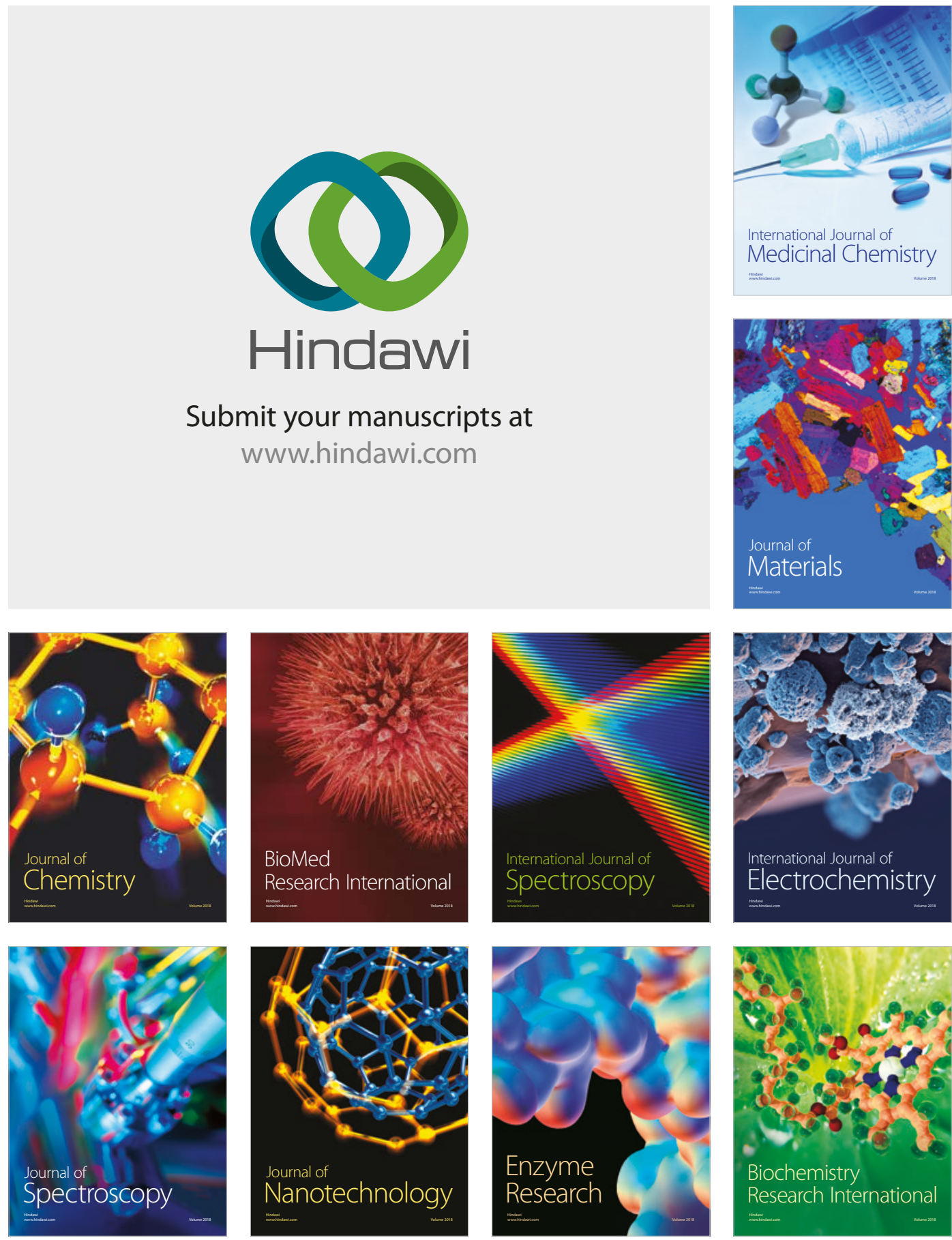
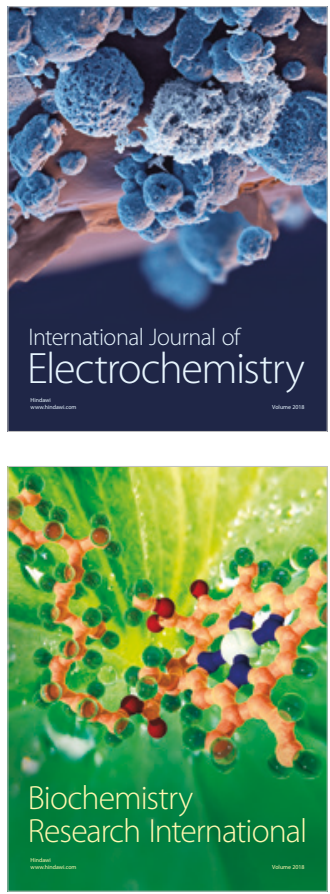